Check for updates

Cite this: Mater. Adv., 2022, 3,3545

Received 9th January 2022,

Accepted 23rd February 2022

DOI: $10.1039 / \mathrm{d} 2 \mathrm{ma} 00024 \mathrm{e}$

rsc.li/materials-advances

\section{A near-infrared intelligent molecular rotor with aggregation induced-emission for viscosity detection of liquids $\dagger$}

\author{
Lingfeng Xu, (D) *ab Yanrong Huang, ${ }^{c}$ Xinkang Peng, ${ }^{a}$ Kui Wu, ${ }^{d}$ Chunfang Huang ${ }^{a}$ \\ and Limin Liu*a
}

\begin{abstract}
Liquid safety has become a widespread issue, due to the potential adverse effects on public health. To the best of our knowledge, viscosity might be a representative physical parameter during the food deterioration process. Monitoring viscosity variation during the food spoilage process is of great importance, thus a convenient inspection technique with rapid detection, high sensitivity, a simple detection process, and fast results output needs to be found. Herein, a near-infrared intelligent molecular rotor (DPADQ) is proposed to describe the micro-viscosity via a fluorescence analytical method with a typical turn-on fluorescence signal. This molecular rotor has a flexible donor- $\pi-a c c e p t o r$ conjugated structure and forms a typical twisted intramolecular charge transfer system. DPADQ features a large Stokes shift (203 nm in the highly viscous glycerol system), longer wavelength emission (725 nm) and typical aggregation-induced emission character. Due to its excellent optical properties, especially the viscosity-sensitive coefficient of 0.55 , a thickening effect with various food gums was determined. And the capability of DPADQ to evaluate the spoilage extent of beverages with viscosity variations as a robust indicator was explored as well. The results indicated that a linear relationship can be found between the viscosity variations and fluorescence fluctuations. This strategy can motivate us to design multi-functional molecular tools for food safety inspection with the use of the fluorescence analytical technique.
\end{abstract}

\section{Introduction}

Spoiled liquids usually cause acute diarrhea, digestion system disorders, and even serious kidney and liver failure, or death, which may cause serious threats to public health. ${ }^{1-3}$ In the field of modern food consumption, fresh liquids, especially fresh pressed beverages, are quite favorably received by customers. However, this kind of beverage is perishable, and usually has a short shelf life at ambient temperature. Even in the refrigerator, fresh liquids may deteriorate in just a few days. With increasing

\footnotetext{
${ }^{a}$ School of Chemistry and Chemical Engineering, Jinggangshan University, Ji'an, Jiangxi 343009, China. E-mail: llm24@126.com

${ }^{b}$ State Key Laboratory of Luminescent Materials \& Devices, Guangdong Provincial Key Laboratory of Luminescence from Molecular Aggregates, College of Materials Science \& Engineering, South China University of Technology, Guangzhou 510640, China.E-mail: rs7lfxu@outlook.com

${ }^{c}$ School of Food Science and Engineering, Guangdong Province Key Laboratory for Green Processing of Natural Products and Product Safety, South China University of Technology, Guangzhou 510640, China

${ }^{d}$ Fine Chemical Industry Research Institute, School of Chemistry, Sun Yat-sen University, Guangzhou 510275, China

$\dagger$ Electronic supplementary information (ESI) available. See DOI: 10.1039/ d2ma00024e
}

awareness of foodborne illness among the public, food safety inspection has become an essential program, especially for the long-term transportation of fresh commercial liquid beverages. ${ }^{4-7}$ During the spoilage process, viscosity of the liquid is an important physical factor, and it is closely related to the spoilage extent; thus, the monitoring of beverage viscosity is necessary. Generally, viscosity determination can be achieved by using various viscometers, including the falling ball viscometer, capillary viscometer, rotating viscometer, etc. ${ }^{8-10}$ or rheometers. ${ }^{11}$ These methods have been designed for macroscopic viscosity measurement, for which complex pretreatment processes and time-consuming detection programs are essential, and large volumes of liquid samples are required. ${ }^{12,13}$ Thereby, an in situ monitoring method for microscopic detection with easy operation, fast result output, less sample required, and high sensitivity is urgently needed.

Among the multiple detection methods, one representative photoluminescence technology, the fluorescence analytical technique, has become increasingly attractive in viscosity sensing, due to its intrinsic advantages such as easy operation, visualization, real-time analysis, rapid result release, and so on. ${ }^{14}$ With the aim of micro-viscosity sensing, to date, many kinds of fluorescent 
molecular rotators (FMRs) have been introduced as sensors with widespread use in biological areas, ${ }^{15-17}$ environmental analytical fields, ${ }^{18,19}$ chemical analysis programs,${ }^{20}$ etc. The biological field has some of the most widely applied FMRs, thus, we selected several representative fluorescence-based viscosity sensing probes for comparison, as shown in Table S1 (ESI $\dagger$ ). It can be seen that most of the reported FMRs not only displayed short emission wavelengths, but also showed short Stokes shifts, which may limit their further application in other areas. In general, molecular rotors are sensitive to viscosity changes, and this is reflected in the signal of fluorescence intensity. Fluorescence phenomena can be produced by the conformations of individual fluorophores via twisted intramolecular-charge transfer (TICT). This is a relatively common phenomenon in the donor- $\pi$-acceptor $(D-\pi-A)$ system, which consists of electron donating and accepting groups, and the linking bond. Such FMRs are usually in a quenched state in a lowviscosity environment, and the energy can be dissipated through non-radiative decay, which leads to free intramolecular rotation, distortion and vibration. In high-viscous media, intramolecular motions are inhibited, and energy is released through radiative decay, resulting in stronger fluorescence. ${ }^{21}$ In the cases of these molecules, the accessibility of the energy charge transfer state is responsible for the emission intensity. And the twisting is viscosity dependent, so viscosity sensing can be achieved by this class of fluorophores, with the existence of the TICT process. On the other hand, most of the current FMRs are composed of large conjugated hydrophobic structures, which prefer to aggregate in aqueous media, leading to fluorescence signal quenching (the typical aggregation caused quenching effect). ${ }^{22}$ In contrast, the FMRs with the aggregation-induced emission (AIE) feature can avoid the quenching effect in the aggregated state, which makes them available to be applied in aqueous media. ${ }^{23}$ So far, several kinds of AIEgens have been developed for food quality monitoring. In the deterioration process, various toxic volatile amines, ${ }^{24,25}$ food allergens, ${ }^{26}$ enzymes, ${ }^{27-29}$ and so on, are considered to be closely related with the spoilage degree of raw foods. However, FMRs with a large Stokes shift, long emission wavelength and AIE feature applicable for liquid food spoilage tracking through viscosity measurement are still lacking.

In this work, an intelligent molecular rotor, DPADQ, with a typical TICT mechanism and AIE character was developed for viscosity sensing. This rotor is constructed by a triphenylamine electron donor (D) group and a quinolinium electron acceptor (A), forming a flexible $\mathrm{D}-\pi-\mathrm{A}$ type rotor through the $\pi$-conjugated linker. This rotor displayed a large Stokes shift not only in distilled water, but also in a glycerol-water system. An obvious 'turn-on' signal occurred at $725 \mathrm{~nm}$ with an increase in viscosity. Moreover, the corresponding sensitivity, selectivity and photo-stability in various commercial beverages were investigated of the rotor DPADQ, and it was found to be applicable in producing a fluorescence signal in beverages. The potentiality of monitoring the thickening effect by adding thickeners with various mass contents was tested, and the results further confirmed its viscosity sensing capacity. Concerning this advantage, this molecular rotor was applied to track the viscosity variations in the beverage spoilage process.
By combining the fluorescence analytical technique and customized synthesis of functional molecules, a linear relationship was established between the viscosity variations and fluorescence fluctuations. Thereby, this work can offer a powerful sensing tool to satisfy the urgent needs of food safety inspection.

\section{Experimental}

\section{Chemical reagents and instruments}

Bromoethane, 4-bromo-2,6-dihydroxybenzaldehyde, diphenylamine, (4-bromophenyl)boronic acid, 4-methylquinoline, palladium acetate $\left(\mathrm{Pd}(\mathrm{OAc})_{2}\right)$, tetrabutylammonium bromide (TBAB), potassium carbonate, tetrahydrofuran (THF), toluene, pyridine, dichloromethane (DCM), dimethylsulfoxide (DMSO), methanol, ethanol, ethyl acetate, acetonitrile, phosphate buffer solution (PBS) and various metal salts, such as sodium chloride, calcium chloride, magnesium sulphate, and so on, were purchased from Shanghai Aladdin Bio-Chem Technology Co., Ltd. Common food additives including beet molasses, sodium benzoate, glucose, disodium hydrogen phosphate, trisodium citrate dehydrate, sodium carboxymethyl cellulose, arabic gum, and xanthan gum were obtained from Bide pharmacy technology (Shanghai) Co., Ltd. All chemical reagents used in this work were of analytical grade.

Nuclear magnetic resonance (NMR) spectra were acquired with a Bruker Avance III HD 600 NMR spectrometer. High resolution mass spectra were obtained with an Agilent 7250 and JEOL-JMS-T100LP AccuTOF mass spectrometer. Fluorescence spectra were recorded by a Hitachi F-7000 fluorescence spectrophotometer. Absorption spectra were recorded on a Hitachi U-3010 UV-vis spectrophotometer. The viscosity determination test was performed on a rotating viscometer (DV2T, Brookfield, Ametek Corp., USA).

\section{Synthesis procedure of the molecular rotor DPADQ}

The detailed synthesis route and corresponding synthesis procedure of the molecular rotor DPADQ are displayed in Scheme S1 (ESI $\dagger$ ) and the Experimental section (see Section S1 in the ESI $\dagger$ ), and the test results are shown in Fig. S1-S15 (ESI $\dagger$ ).

\section{Measurements of optical properties}

According to a previous study, ${ }^{30}$ the molecular rotor DPADQ was dissolved in DMSO to prepare a stock solution with a concentration of $1 \mathrm{mM}$ that was stored in the dark at a temperature of $3{ }^{\circ} \mathrm{C}$. During the test procedure, the concentration of DPADQ was controlled as $10 \mu \mathrm{M}$. The viscosity determination procedure was as follows. The probe was added into a $3 \mathrm{~mL}$ solution consisting of various volume percentages of distilled water and glycerol (from $0 \%$ to $99 \%$, including 1\% DMSO), and the fluorescence spectra and UV-vis absorption were recorded in this mixture system. Corresponding viscosity values were measured and recorded by the viscometer. The AIE property of the molecular rotor DPADQ was studied in DMSO/water mixtures with different water contents (from $0 \%$ to $90 \%$ ). Solvents with various polarities, such as ethanol, methanol, DMSO, etc. were selected to test the emission properties of the molecular rotor 
DPADQ in a complex solvent environment. DPADQ was added into selected solvents and shaken before the test. In the specificity test, solutions with various potential interfering analytes (including $\mathrm{K}_{2} \mathrm{CO}_{3}, \mathrm{NaCl}$, glucose, and so on) were prepared with distilled water, and DPADQ was added into each solution. The resulting solutions were mixed uniformly before the spectra were recorded. In the temperature effect experiment, DPADQ was added into glycerol containing $1 \%$ DMSO at different temperatures, such as normal body temperature $\left(37^{\circ} \mathrm{C}\right)$, fresh-maintenance temperature $\left(5{ }^{\circ} \mathrm{C}\right)$ and common room temperature $\left(25^{\circ} \mathrm{C}\right)$. For all measurements, mixed solutions were transferred to a quartz cell, absorption spectra were recorded from $400 \mathrm{~nm}$ to $750 \mathrm{~nm}$, and emission spectra were recorded from $500 \mathrm{~nm}$ to $900 \mathrm{~nm}$. The excitation wavelength was set as $500 \mathrm{~nm}$.

\section{Fluorescence analysis of the thickening effect}

Three kinds of food thickeners, sodium carboxymethyl cellulose, arabic gum, and xanthan gum with various mass concentrations (from $1 \mathrm{~g} \mathrm{~kg}^{-1}$ to $5 \mathrm{~g} \mathrm{~kg}^{-1}$ ), were dissolved in twice-distilled water, followed by the addition of DPADQ $(10 \mu \mathrm{M})$. Before the test, the mixtures were treated with ultrasound for $10 \mathrm{~min}$ to eliminate bubbles in the high-viscous solution, and left at room temperature for $1 \mathrm{~h}$.

\section{Viscosity tracking during the liquid spoilage process}

Three kinds of commercial liquid beverages, pear juice, grape juice and pomelo juice, were stored at different temperatures $\left(25{ }^{\circ} \mathrm{C}\right.$ and $\left.5{ }^{\circ} \mathrm{C}\right)$ for 9 days. The fluorescence emission spectra were recorded at different time intervals (at day 0 , day 3, day 6 , and day 9$)$ with the addition of DPADQ $(10 \mu \mathrm{M})$.

The relationship between the fluorescence intensity enhancement and viscosity variation was fitted into the linear equation: $\left(\eta_{n}-\eta_{0}\right) / \eta_{0}-\left(F_{n}-F_{0}\right) / F_{0}$, in which $\eta_{n}$ and $\eta_{0}$ were defined as the viscosity of the beverages at day 0 and day $n(0<$ $n<10)$, and $F_{0}$ and $F_{n}$ were the fluorescence intensity of beverages at day 0 and day $n(0<n<10)$.

\section{Results and discussion}

\section{Molecular rotor design and synthesis}

When designing an intelligent molecular rotor for viscosity determination, several rotatable parts should be introduced into the chemical structure to perceive microenvironment viscosity variation. ${ }^{31}$ Ideally, stronger fluorescence can be achieved in more viscous liquids, and weaker signals will be emitted in less viscous liquids, forming a viscosity-dependent fluorescence response. Usually, in terms of a representative donor group, triphenylamine and its corresponding derivatives commonly appear in the probe design procedure due to their strong electron donating capacity and multiple rotatable benzene rings, especially when connected with another conjugated structure in a further step. ${ }^{32}$ On the other side, the typical acceptor groups of quinoline and its derivatives (especially quinolinium) have attracted much attention in recent studies due to their strong electron withdrawing capacity and excellent photophysical
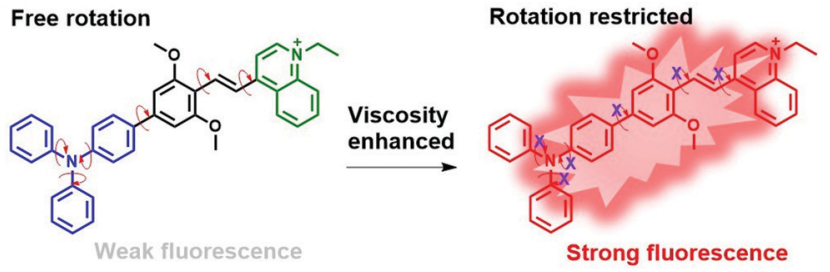

Scheme 1 Detailed viscosity sensing mechanism of the rotor DPADQ.

properties. $^{33,34}$ Based on these characterizations, two moieties, a triphenylamine donor and quinolinium acceptor, were linked through a conjugated linker, forming a typical TICT system. This FMR can undergo intramolecular D-A twisting around a single bond, and conversion between a locally excited state and stabilized ICT state, holding great promise in viscosity sensing. ${ }^{35}$ Dimethyl ether benzene was chosen to extend the conjugated system, which can improve the fluorescence performance. And dimethyl ether was introduced to enhance the electron-donating capacity, which can further increase the emission wavelength to some extent. The viscosity detection mechanism is displayed in Scheme 1 . The rotatable parts, including the benzene rings and $\pi$ bridge (carbon-carbon double bond), can rotate freely in lowviscous media, resulting in a non-radiative quenching of the excited state. In contrast, in a highly viscous environment, rotation was restricted, thus the fluorescence signal was dramatically enhanced via a radiative transition pathway. ${ }^{36}$ Thereby, a 'turn-on' intelligent fluorescent tool was constructed for viscosity determination. This molecular rotor can be readily synthesized in five steps, as shown in Scheme S1 (ESI $\dagger$ ). In detail, the first step was the substitution of phenolic hydroxyl groups, and the second step was the preparation of a donor group triphenylamine derivative. In the third step, the preparation underwent a Suzuki coupling reaction procedure, then the acceptor group quinolinium was obtained via a salification reaction. Finally, following a dehydration condensation reaction between the D and A moieties, the molecular rotor DPADQ was obtained.

\section{Optical properties of the rotor DPADQ}

The fluorescence response toward viscosity determination was investigated at first. The spectral properties of the rotor DPADQ in distilled water and 99\% glycerol were studied. As displayed in Fig. 1a and b, a weak fluorescence signal was observed in the distilled water, which was due to the free rotation of aromatic rings and the energy being consumed by non-radiative pathways. In contrast, a stronger fluorescence signal at $725 \mathrm{~nm}$ was emitted in the glycerol system, since the aromatic ring rotation was inhibited, and the radiative pathway was restored. Thus, this rotor can be applied to discriminate high-viscous media from low-viscous ones using the fluorescence method. Furthermore, absorption spectra in distilled water and glycerol were recorded. A slight red shift was found in the absorption peak of glycerol, which may be attributed to an enlargement of conjugation degree with the parallel stacking of DPADQ in a highly viscous microenvironment. ${ }^{37}$ A large Stokes shift is capable of avoiding the interference of excitation light, and the signal-to-noise ratio 

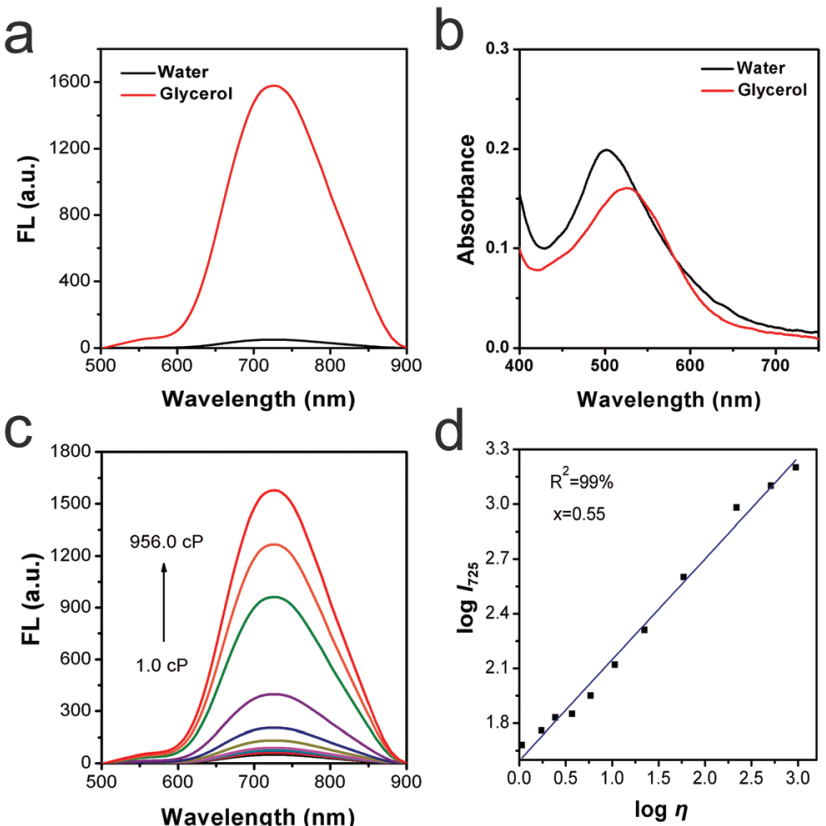

Fig. 1 (a) Fluorescence spectra of the rotor DPADQ $(10 \mu \mathrm{M})$ in water and glycerol. (b) Absorption spectra of the rotor DPADQ $(10 \mu \mathrm{M})$ in water and glycerol. (c) Fluorescence emission spectra of the molecular rotor DPADQ $(10 \mu \mathrm{M})$ with increasing viscosity in water/glycerol mixtures (glycerol volume fraction: $0-99 \%$ ). (d) The linear relationship of $\log l_{725}$ and $\log \eta$. $\lambda_{\mathrm{ex}}=500 \mathrm{~nm}, \lambda_{\mathrm{em}}=725 \mathrm{~nm}$

can be enhanced as well. The Stokes shift in distilled water and glycerol was investigated, respectively. As shown in Fig. S16 $(\mathrm{ESI} \dagger)$, the Stokes shift was found to be $235 \mathrm{~nm}$ in distilled water, and $203 \mathrm{~nm}$ in the glycerol. These results confirmed the potential capability of viscosity determination and superior photophysical properties of the rotor DPADQ.

Next, the viscosity-responsive optical performance was investigated in more detail with a water-glycerol system with various glycerol fractions (from 0-99\%). As displayed in Fig. 1c, with higher proportions of glycerol in the water/glycerol mixture system, the fluorescence signal was enhanced gradually, due to the forbidden intramolecular rotation between the D and A groups, and finally reached about 32 -fold at $725 \mathrm{~nm}$ at maximum. On the other hand, a good linear relationship between the fluorescence intensity $\left(\log I_{725}\right)$ and the viscosity $(\log \eta)$ was found by fitting the Förster-Hoffmann equation (see Section S2 in the ESI $\dagger$ ), with $R^{2}=$ 0.99 and $x=0.55$. These results demonstrated that the rotor DPADQ can respond to viscosity variations, which indicated that it can be instrumental in viscosity tracking as an intelligent rotor.

In addition, the AIE property of the rotor DPADQ was investigated in a DMSO/water system where the water fraction $\left(f_{\mathrm{w}}\right)$ was increased from $0 \%$ to $90 \%$. The detailed testing results are presented in Fig. S17 (ESI $\dagger$ ). It was found that the signal was rather weak when $f_{\mathrm{w}}$ was below $60 \%$, while the fluorescence intensity increased sharply with a higher $f_{\mathrm{w}}$ exceeding $60 \%$, and it reached nearly 30 -fold in the mixed solution with $f_{\mathrm{w}}=90 \%$. The typical AIE feature was observed.

Next, to verify the stability of the rotor DPADQ in liquid media with different polarities, DPADQ was dissolved in
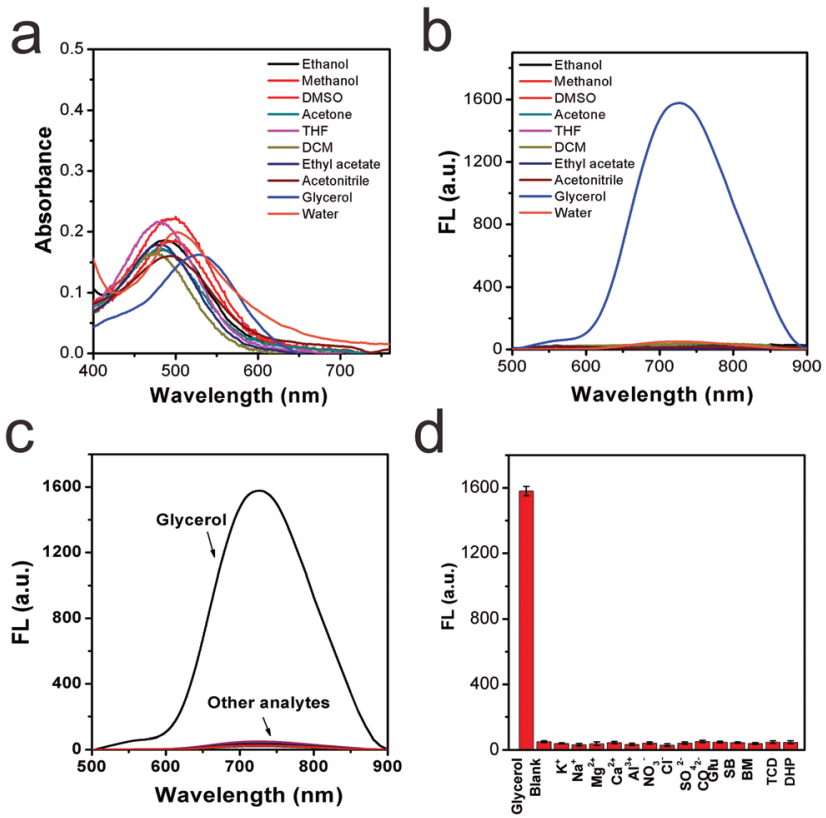

Fig. 2 (a) Absorption spectra of the molecular rotor DPADQ $(10 \mu \mathrm{M})$ in various solvents. (b) Fluorescence spectra of the molecular rotor DPADQ $(10 \mu \mathrm{M})$ in various solvents. (c) Fluorescence spectra of the rotor DPADQ $(10 \mu \mathrm{M})$ in glycerol $(99 \%)$ and in the absence or presence of other potential analytes $(100 \mu \mathrm{M})$ in water, including $\mathrm{K}^{+}, \mathrm{Na}^{+}, \mathrm{Mg}^{2+}, \mathrm{Ca}^{2+}, \mathrm{Al}^{3+}, \mathrm{NO}_{3}{ }^{-}, \mathrm{Cl}^{-}$, $\mathrm{SO}_{4}^{2-}, \mathrm{CO}_{3}^{2-}$, glucose (Glu), sodium benzoate (SB), beet molasses (BM), trisodium citrate dehydrate (TCD), and disodium hydrogen phosphate (DHP). (d) Fluorescence intensity of the rotor DPADQ $(10 \mu \mathrm{M})$ at $725 \mathrm{~nm}$ in glycerol (99\%) and in the presence of various potentially interfering substances $(100 \mu \mathrm{M})$ in water, respectively. $\lambda_{\mathrm{ex}}=500 \mathrm{~nm}$.

ordinary solvents with different polarities, including ethanol, methanol, DMSO, acetone, THF, DCM, ethyl acetate, and acetonitrile, and glycerol was set as the control. As shown in Fig. 2a, the absorption peak was around $500 \mathrm{~nm}$ in most of the ordinary solvents, while the differences can be ignored. In contrast, a small red shift (about $32 \mathrm{~nm}$ ) was found in the absorption peak of glycerol. This phenomenon was due to the parallel stacking structure formed in a highly viscous medium, thus the conjugation was extended, causing a red shift of the absorption spectrum. ${ }^{38}$ As presented in Fig. $2 \mathrm{~b}$, the emission intensity in the above various solvents remained extremely weak and nearly unchanged, while a strong fluorescence signal was released in the glycerol. Detailed photo-physical properties of the rotor DPADQ in these solvents are shown in Table S2 (ESI $\dagger$ ). The corresponding quantum yields in various solvents were investigated as well, as displayed in Table S3 (ESI $\dagger$ ). The results demonstrated that the fluorescence variation of the rotor DPADQ was merely a response to viscosity changes even in complex solvents. On the other hand, the stability at different $\mathrm{pH}$ values was evaluated. The rotor DPADQ was dissolved in PBS with different $\mathrm{pH}$ values from 3.0 to 9.0, wherein the final concentration of DPADQ was $10 \mu \mathrm{M}$. It was found that the fluorescence intensity remained stable in this $\mathrm{pH}$ value range, the fluctuation was rather small, as shown in Fig. S18 (ESI $\dagger$ ). It was then necessary to study the selectivity, since various food additives are added to improve the texture and nutritional value 
of beverages. ${ }^{39}$ Based on this consideration, several kinds of potentially interfering inorganic salt analytes including $\mathrm{K}^{+}, \mathrm{Na}^{+}$, $\mathrm{Mg}^{2+}$, etc., common food additives, such as glucose (Glu), sodium benzoate (SB), beet molasses (BM), trisodium citrate dehydrate (TCD), etc., and glycerol were added to the solution and tested using the fluorescence method. As displayed in Fig. $2 \mathrm{c}$ and d, the fluorescence signal was relatively weak in the presence of all these species, except the glycerol group, which further confirmed that the rotor DPADQ had the potential capability of viscosity sensing in a complex medium environment.

It is well known that a lower temperature can delay the deterioration of liquids, especially in cold chain transportation and the food storage industry, ${ }^{40,41}$ and the viscosity is usually enhanced and may improve the drinking texture. In contrast, a higher temperature may be helpful in increasing the flowability toward food processing. Thus, the viscosity of glycerol at different temperatures was tested using the fluorescence method. Glycerol solutions (containing 1\% DMSO) were placed in a freshmaintenance layer in the refrigerator, at ambient temperature, and at $37{ }^{\circ} \mathrm{C}$ in a water bath, respectively. The corresponding fluorescence inspection was performed after the glycerol was stored at different temperatures for one hour, as shown in Fig. S19 (ESI $\dagger)$. The fluorescence intensity increased as the temperature reduced, displaying a sensitivity toward temperature variation. Indeed, the viscosity of glycerol at a higher storage temperature reduced, whereas the viscosity increased at a lower storage temperature. This phenomenon was confirmed by the viscometer. Overall, all the optical detection results indicated that the molecular rotor DPADQ can be appropriately applied as a viscosity identification tool.

\section{Viscosity detection of thickening effect}

In order to enhance the consistency, homogeneity and stability, with the slippery and palatable taste, several kinds of food thickeners can be found in commercial beverages. ${ }^{42}$ Thus, three kinds of food thickeners, sodium carboxymethyl cellulose, arabic gum, and xanthan gum, were dissolved in distilled water, respectively. The viscosity variations were determined using the fluorescence method. As shown in Fig. 3a, c and e, the emission intensity was found to increase after adding different amounts of thickeners ranging from $1 \mathrm{~g} \mathrm{~kg}^{-1}$ to $5 \mathrm{~g} \mathrm{~kg}^{-1}$, positively correlating with the content of the thickener. Meanwhile, a good linear relationship between the mass concentrations of food thickeners and corresponding fluorescence intensities was observed, as shown in Fig. 3b, d and f. From the fitted linear slope, we found that the thickening efficiency of each food thickener was totally different. Specifically, xanthan gum displayed the highest thickening efficiency among these three thickeners, while sodium carboxymethyl cellulose had the lowest thickening efficiency. In this case, the viscosity fluctuation caused by the extra addition of thickeners can be evaluated by this intelligent rotor tool, which may be beneficial for thickener selection in beverage preparation.

\section{Viscosity tracking in the spoilage process}

Viscosity plays an important role in commercial liquids; in particular, sustainable nutrition should be maintained during
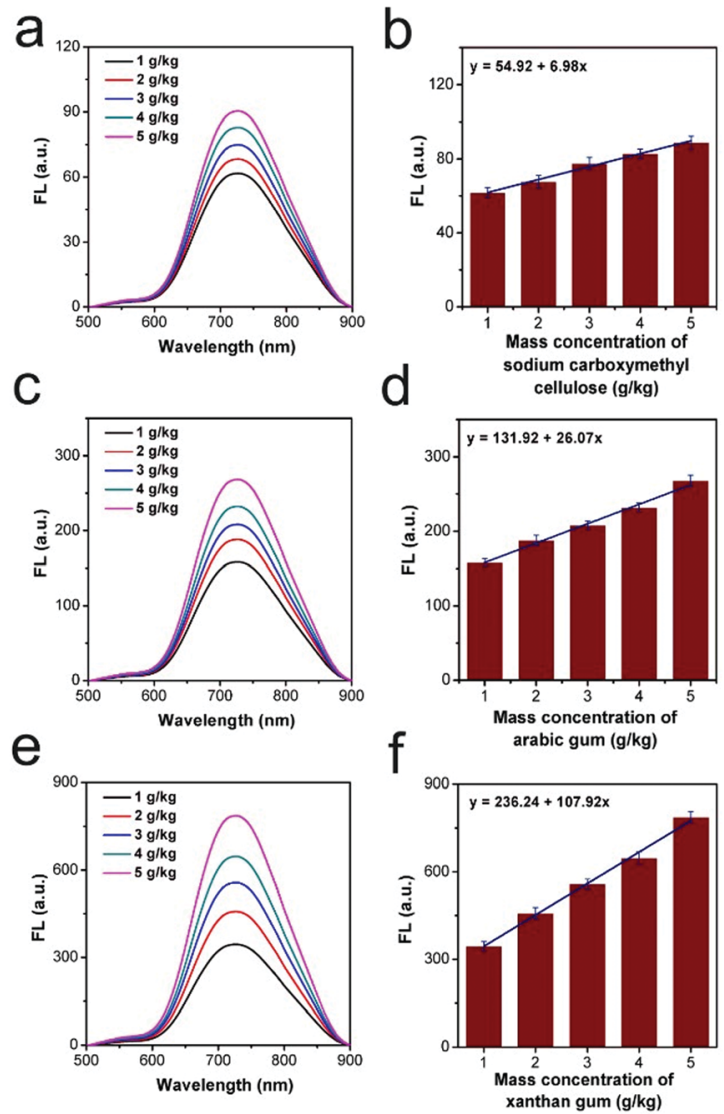

Fig. 3 (a) Fluorescence spectra of the rotor DPADQ $(10 \mu M)$ in the presence of different mass contents of sodium carboxymethyl cellulose in water. (b) Linear fitting relationship of corresponding fluorescence intensities of the rotor DPADQ $(10 \mu \mathrm{M})$ at $725 \mathrm{~nm}$ with various mass contents of sodium carboxymethyl cellulose in water. (c) Fluorescence spectra of the rotor DPADQ $(10 \mu \mathrm{M})$ in the presence of different mass contents of arabic gum in water. (d) Linear fitting relationship of corresponding fluorescence intensities of the rotor DPADQ $(10 \mu \mathrm{M})$ at $725 \mathrm{~nm}$ with various mass contents of arabic gum in water. (e) Fluorescence spectra of the rotor DPADQ $(10 \mu \mathrm{M})$ in the presence of different mass contents of xanthan gum in water. (f) Linear fitting relationship of corresponding fluorescence intensities of the rotor DPADQ $(10 \mu \mathrm{M})$ at $725 \mathrm{~nm}$ with various mass contents of xanthan gum in water.

the long-distance transportation and shop storage period, in order to satisfy the instant selling demands of fresh liquids for the consumer. Thus, to ensure food safety, auxiliary detection means need to be developed. Herein, we selected nine commercial beverages, pear juice, kumquat juice, watermelon juice, pomelo juice, grape juice, fruit vinegar, edible oil, sweet milk and yogurt, as research objects, and distilled water acted as the control group. First, the photostability of the rotor DPADQ in these beverages was assessed. The corresponding fluorescence signals in these beverages under continuous light irradiation for 120 min remained relatively stable, and the results indicated that this rotor DPADQ can be applied universally in various beverages, as shown in Fig. S20 (ESI $\dagger$ ). Subsequently, the corresponding viscosities of these beverages were investigated as well, as presented in Fig. S21 (ESI $\dagger$ ). The fluorescence intensities in these beverages were completely different, which 
was attributed to the differences of internal viscosity among these beverages. Moreover, the viscosities of these beverages were measured in detail by a viscometer, respectively (as shown in Table S4, ESI $\dagger$ ). It can be found that a positive relationship existed between the viscosity value and fluorescence intensity. Indeed, the fluorescence intensity fluctuated with the viscosity change, indicating that the rotor DPADQ can serve as an intelligent monitoring tool in viscosity determination in various beverages.

Based on this, the spoilage process was monitored using the fluorescence method, and the tracking potential of this rotor was explored. Three representative beverages, pear juice, grape juice and pomelo juice, were selected herein. These beverages were stored at ambient temperature (about $25{ }^{\circ} \mathrm{C}$ ) and a lower temperature (fresh-maintenance temperature around $5{ }^{\circ} \mathrm{C}$ ) for 9 days, respectively. The observed phenomena during the storage process at different temperatures were recorded by a digital camera and fluorescence spectrometer with the rotor DPADQ. As shown in Fig. 4a, the selected beverages gradually became turbid and unclear, floating objects were even observed after 6 days, when the beverages were stored at ambient temperature, especially in the pomelo juice. During the spoiling process, the fluorescence intensities increased obviously to some extent in these beverages. By comparison, as shown in Fig. $4 \mathrm{~b}$, a relatively transparent liquid could be observed after being stored at the fresh-maintenance temperature in the refrigerator, and the fluorescence intensity was enhanced in a limited range. These results demonstrated that the spoilage process can be tracked with the rotor DPADQ using the fluorescence method. This study also revealed that the deterioration phenomenon was much worse at ambient temperature, whereas a lower temperature can prolong the preservation time of liquids.
Next, the viscosity increase and corresponding fluorescence intensity enhancement of these beverages were recorded at different intervals within 9 days quantitatively. As presented in Fig. 5a, c and e, when stored at ambient temperature, the viscosity of the pear juice, grape juice and pomelo juice increased by $58.1 \%, 46.3 \%$ and $89.4 \%$, respectively, within 9 days. In contrast, the viscosity of the pear, grape and pomelo juices increased by $23.9 \%, 16.6 \%$ and $43.5 \%$ when stored at the lower temperature (fresh-maintenance layer) over 9 days. From the fluorescence perspective, it is noteworthy that the fluorescence intensities of the pear, grape and pomelo juices increased by $29.3 \%, 24.7 \%$ and $45.9 \%$ when the beverages were stored at ambient temperature for 9 days. In comparison, the fluorescence intensity only enhanced by $17.2 \%, 13.1 \%$ and $23.5 \%$ in the fresh-maintenance layer of the refrigerator. The results presented in Fig. 5b, $\mathrm{d}$ and $\mathrm{f}$ indicate that the variation range in the fluorescence intensity was relatively narrower than that at ambient temperature, which was consistent with the viscosity fluctuation results. Following this, we estimated that a similar increment relationship existed between the viscosity value and fluorescence intensity. As displayed in Fig. 5g, a fitted linear relationship can be found between the viscosity increment percentage $\left(\eta_{n}-\eta_{0}\right) / \eta_{0} \times 100 \%$ and the fluorescence intensity increment percentage $\left(F_{n}-F_{0}\right) / F_{0} \times 100 \%$. Accordingly, we have successfully proved that the rotor DPADQ can act as an intelligent monitoring tool to visualize the liquid spoilage process via the fluorescence technique.

\section{Conclusions}

In summary, an intelligent molecular rotor, DPADQ, was designed and systematically synthesized successfully. A corresponding

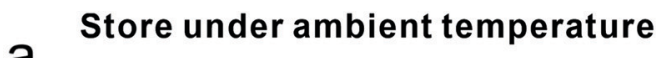

a
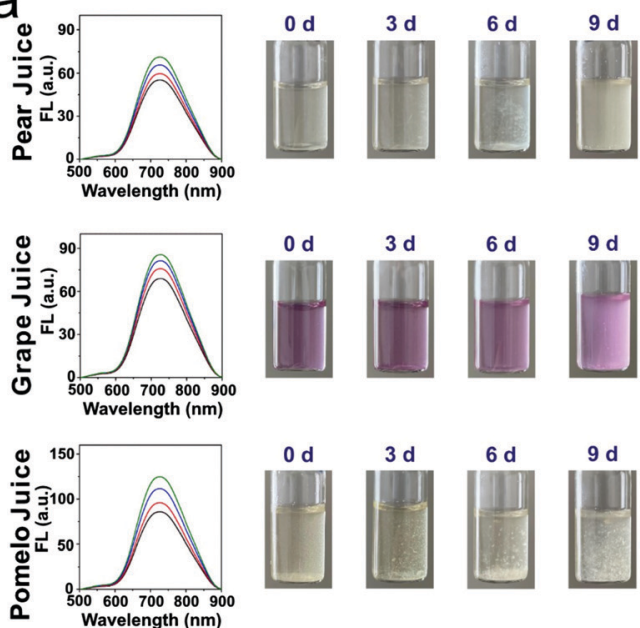

$b$
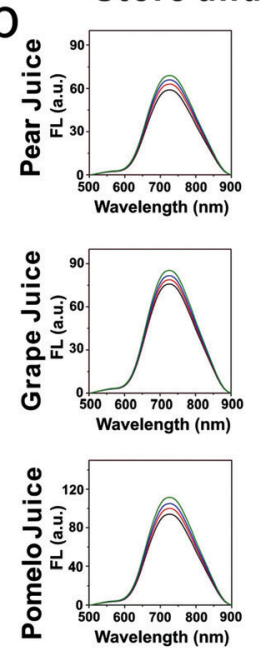

Store under lower temperature
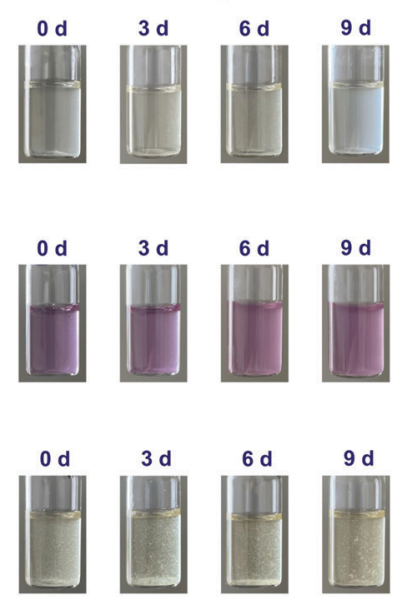

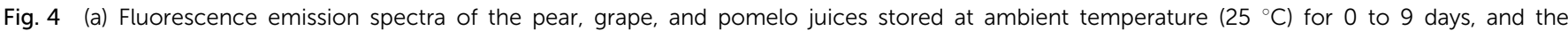

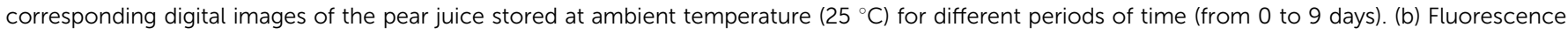

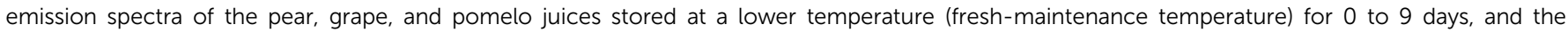

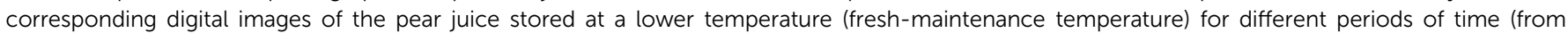
0 to 9 days). 

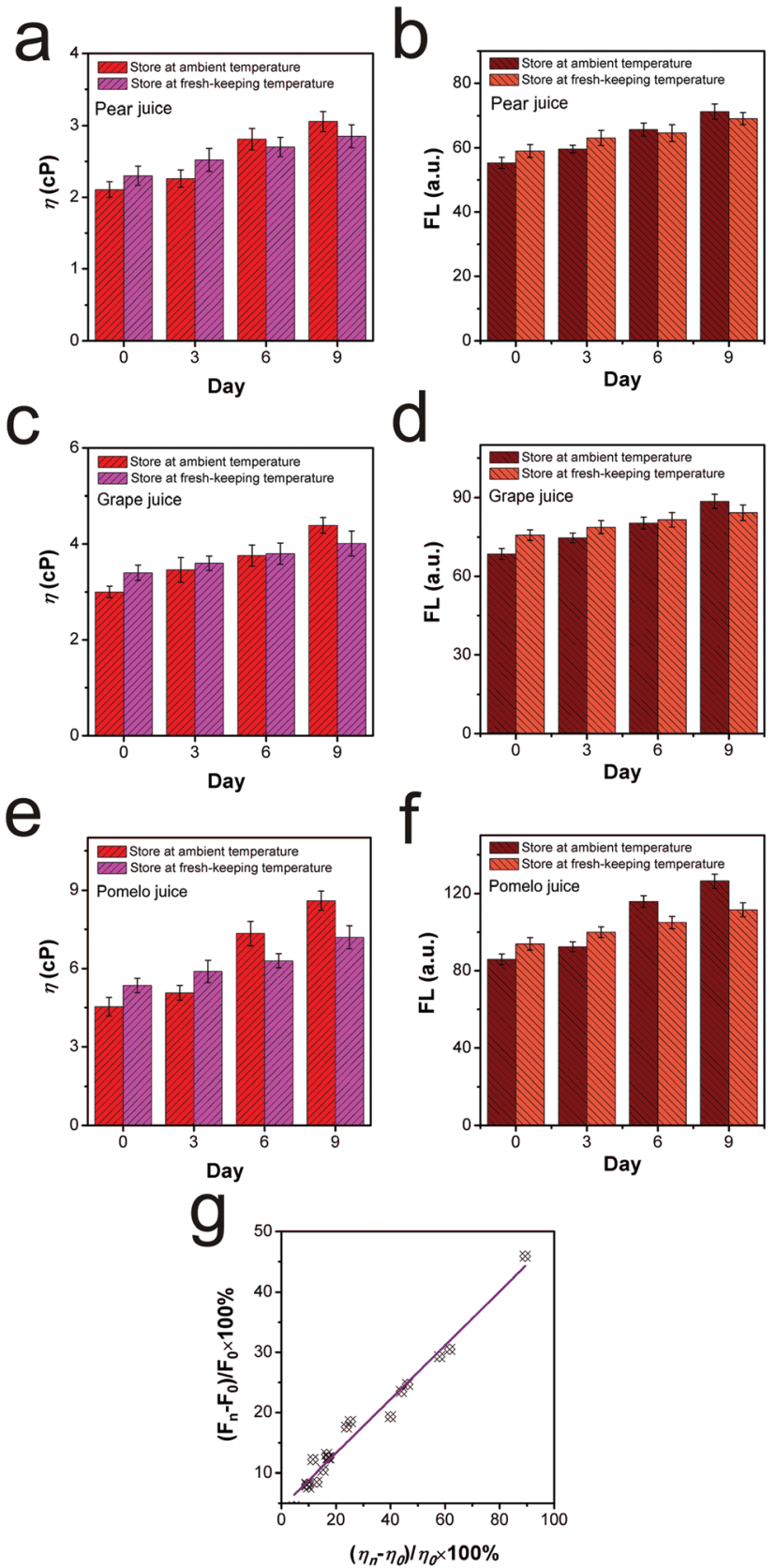

Fig. 5 (a) Viscosity values of the pear juice stored at ambient temperature and fresh-maintenance temperature over 9 days. (b) Fluorescence intensities of the rotor DPADQ $(10 \mu \mathrm{M})$ at $725 \mathrm{~nm}$ in the pear juice stored at ambient temperature and fresh-maintenance temperature over 9 days. (c) Viscosity values of the grape juice stored at ambient temperature and fresh-maintenance temperature over 9 days. (d) Fluorescence intensities of the rotor DPADQ $(10 \mu \mathrm{M})$ at $725 \mathrm{~nm}$ in the grape juice stored at ambient temperature and fresh-maintenance temperature over 9 days. (e) Viscosity values of the pomelo juice stored at ambient temperature and fresh-maintenance temperature over 9 days. (f) Fluorescence intensities of the rotor DPADQ $(10 \mu \mathrm{M})$ at $725 \mathrm{~nm}$ in the pomelo juice stored at ambient temperature and fresh-maintenance temperature over 9 days. (g) Fitted linear relationship between the fluorescence intensity enhancement percentage and viscosity value increment percentage.

investigation of viscosity sensing capability was performed. Excellent sensitivity, selectivity and photostability were found not only in the glycerol-water system, but also in commercial beverages. Afterward, we utilized the rotor to characterize a thickening effect achieved by adding common food thickeners. The results confirmed the rotor's potential to be applied in a liquid thickening program. Furthermore, to obtain a better understanding of the spoilage process, the viscosity of liquid beverages was tracked using the molecular rotor DPADQ and the fluorescence analytical technique. A linear relationship was established between the viscosity variations and fluorescence fluctuations. Such a unique strategy shows that DPADQ is suitable for evaluating the extent of beverage deterioration. This study can motivate us to develop more powerful tools combining molecular fluorescence visualization technology for liquid safety inspection.

\section{Author contributions}

Lingfeng Xu: conceptualization, data curation, writing the original draft. Yanrong Huang: investigation, formal analysis. Xinkang Peng: validation, visualization. Kui Wu: resources, methodology. Chunfang Huang: funding acquisition, writing review \& editing. Limin Liu: supervision, project administration.

\section{Conflicts of interest}

There are no conflicts to declare.

\section{Acknowledgements}

This work was supported by the National Natural Science Foundation of China (21765011, 21808247), Natural Science Foundation of Jiangxi Province (20212BAB214031), Natural Science Foundation of Guangdong Province (2020A1515010610), Doctoral Research Foundation of Jinggangshan University (JZB2006), and Science and Technology Program of Jiangxi Provincial Education Bureau (GJJ211032, GJJ160736).

\section{References}

1 T. King, M. Cole, J. M. Farber, G. Eisenbrand, D. Zabaras, E. M. Fox and J. P. Hill, Food safety for food security: relationship between global megatrends and developments in food safety, Trends Food Sci. Technol., 2017, 68, 160-175.

2 U. Mc Carthy, I. Uysal, R. Badia-Melis, S. Mercier, C. O’Donnell and A. Ktenioudaki, Global food security-issues, challenges and technological solutions, Trends Food Sci. Technol., 2018, 77, 11-20.

3 J. Yates, M. Deeney, H. B. Rolker, H. White, S. Kalamatianou and S. Kadiyala, A systematic scoping review of environmental, food security and health impacts of food system plastics, Nat. Food, 2021, 2, 80-87.

4 Y. Han, W. Yang, X. Luo, X. He, H. Zhao, W. Tang, T. Yue and $\mathrm{Z}$. Li, Carbon dots based ratiometric fluorescent sensing platform for food safety, Crit. Rev. Food Sci. Nutr., 2020, 1-17, DOI: 10.1080/10408398.2020.1814197. 
5 X. Luo, Y. Han, X. Chen, W. Tang, T. Yue and Z. Li, Carbon dots derived fluorescent nanosensors as versatile tools for food quality and safety assessment: A review, Trends Food Sci. Technol., 2020, 95, 149-161.

6 M. Lv, Y. Liu, J. Geng, X. Kou, Z. Xin and D. Yang, Engineering nanomaterials-based biosensors for food safety detection, Biosens. Bioelectron., 2018, 106, 122-128.

7 S. Wibowo, C. Buvé, M. Hendrickx, A. Van Loey and T. Grauwet, Integrated science-based approach to study quality changes of shelf-stable food products during storage: A proof of concept on orange and mango juices, Trends Food Sci. Technol., 2018, 73, 76-86.

8 N. Mäkelä, O. Brinck and T. Sontag-Strohm, Viscosity of $\beta$-glucan from oat products at the intestinal phase of the gastrointestinal model, Food Hydrocol., 2020, 100, 105422.

9 F. Morreale, R. Garzón and C. M. Rosell, Understanding the role of hydrocolloids viscosity and hydration in developing gluten-free bread. A study with hydroxypropylmethylcellulose, Food Hydrocolloids, 2018, 77, 629-635.

10 J. Nsor-Atindana, H. Douglas Goff, W. Liu, M. Chen and F. Zhong, The resilience of nanocrystalline cellulose viscosity to simulated digestive processes and its influence on glucose diffusion, Carbohydr. Polym., 2018, 200, 436-445.

11 X. Chai, Z. Meng and Y. Liu, Comparation of micro-viscosity of liquid oil in different colloidal fat crystal networks using molecular rotors, Food Chem., 2020, 317, 126382.

12 B. Chen, S. Mao, Y. Sun, L. Sun, N. Ding, C. Li and J. Zhou, A mitochondria-targeted near-infrared fluorescent probe for imaging viscosity in living cells and a diabetic mice model, Chem. Commun., 2021, 57, 4376-4379.

13 Y.-F. Wei, X.-F. Weng, X.-L. Sha, R. Sun, Y.-J. Xu and J.-F. Ge, Simultaneous imaging of lysosomal and mitochondrial viscosity under different conditions using a NIR probe, Sens. Actuators, B, 2021, 326, 128954.

14 J.-T. Hou, K.-K. Yu, K. Sunwoo, W. Y. Kim, S. Koo, J. Wang, W. X. Ren, S. Wang, X.-Q. Yu and J. S. Kim, Fluorescent imaging of reactive oxygen and nitrogen species associated with pathophysiological processes, Chem, 2020, 6, 832-866.

15 B. Chen, C. Li, J. Zhang, J. Kan, T. Jiang, J. Zhou and H. Ma, Sensing and imaging of mitochondrial viscosity in living cells using a red fluorescent probe with a long lifetime, Chem. Commun., 2019, 55, 7410-7413.

16 X. Dai, B. Dong, M. Ren and W. Lin, Unique $D-\pi-A-\pi-D$ type fluorescent probes for the two-photon imaging of intracellular viscosity, J. Mater. Chem. B, 2018, 6, 381-385.

17 M. Ren, K. Zhou, L. Wang, K. Liu and W. Lin, Construction of a ratiometric two-photon fluorescent probe to monitor the changes of mitochondrial viscosity, Sens. Actuators, B, 2018, 262, 452-459.

18 M. A. Haidekker and E. A. Theodorakis, Environment-sensitive behavior of fluorescent molecular rotors, J. Biol. Eng., 2010, 4, 11.

19 B. Sk, S. Khodia and A. Patra, T and V-shaped donoracceptor-donor molecules involving pyridoquinoxaline: large Stokes shift, environment-sensitive tunable emission and temperature-induced fluorochromism, Chem. Commun., 2018, 54, 1786-1789.
20 X. Han, F. Hu, W. Chi, X. Ma, S. H. Liu, X. Liu and J. Yin, Unusual intermolecular charge transfer enables supramolecular fluorescent viscosity sensors, Sens. Actuators, B, 2018, 277, 55-61.

21 X. Liu, W. Chi, Q. Qiao, S. V. Kokate, E. P. Cabrera, Z. Xu, X. Liu and Y.-T. Chang, Molecular mechanism of viscosity sensitivity in BODIPY rotors and application to motionbased fluorescent sensors, ACS Sens., 2020, 5, 731-739.

22 F. Würthner, Aggregation-induced emission (AIE): a historical perspective, Angew. Chem., Int. Ed., 2020, 59, 14192-14196.

23 G. Niu, R. Zhang, X. Shi, H. Park, S. Xie, R. T. K. Kwok, J. W. Y. Lam and B. Z. Tang, AIE luminogens as fluorescent bioprobes, TrAC, Trends Anal. Chem., 2020, 123, 115769.

24 J. Hou, J. Du, Y. Hou, P. Shi, Y. Liu, Y. Duan and T. Han, Effect of substituent position on aggregation-induced emission, customized self-assembly, and amine detection of donor-acceptor isomers: implication for meat spoilage monitoring, Spectrochim. Acta, Part A, 2018, 205, 1-11.

25 J. Han, Y. Li, J. Yuan, Z. Li, R. Zhao, T. Han and T. Han, To direct the self-assembly of AIEgens by three-gear switch: morphology study, amine sensing and assessment of meat spoilage, Sens. Actuators, B, 2018, 258, 373-380.

26 Z. Li, L. Wang, W. Guan, C. Ding, Z. Yuan and C. Lu, A novel homolateral and dicationic AIEgen for the sensitive detection of casein, Analyst, 2019, 144, 3635-3642.

27 X. Wei, Q. Wu, Y. Feng, M. Chen, S. Zhang, M. Chen, J. Zhang, G. Yang, Y. Ding, X. Yang, Q. Ye, Y. Zhang, Q. Gu, J. Wang, S. Wu, R. Pang and Y. Li, Off-on fluorogenic substrate harnessing ESIPT and AIE features for in situ and long-term tracking of $\beta$-glucuronidase in Escherichia coli, Sens. Actuators, B, 2020, 304, 127242.

28 Y. Li, Q. Du, X. Zhang, H. Cao and Y. Huang, Kojic acid capped gold nanoclusters with aggregation-induced emission for fluorometric screening of the activity of alkaline phosphatase, Microchim. Acta, 2019, 186, 577.

29 M. Zhao, Y. Gao, S. Ye, J. Ding, A. Wang, P. Li and H. Shi, A light-up near-infrared probe with aggregation-induced emission characteristics for highly sensitive detection of alkaline phosphatase, Analyst, 2019, 144, 6262-6269.

30 Y. Cai, C. Gui, K. Samedov, H. Su, X. Gu, S. Li, W. Luo, H. H. Y. Sung, J. W. Y. Lam, R. T. K. Kwok, I. D. Williams, A. Qin and B. Z. Tang, An acidic pH independent piperazine-TPE AIEgen as a unique bioprobe for lysosome tracing, Chem. Sci., 2017, 8, 7593-7603.

31 Y. Ma, Y. Zhao, R. Guo, L. Zhu and W. Lin, A near-infrared emission fluorescent probe with multi-rotatable moieties for highly sensitive detection of mitochondrial viscosity in an inflammatory cell model, J. Mater. Chem. B, 2018, 6, 6212-6216.

32 J. Yin, X. Kong and W. Lin, Noninvasive cancer diagnosis in vivo based on a viscosity-activated near-infrared fluorescent probe, Anal. Chem., 2021, 93, 2072-2081.

33 Y. Deng and G. Feng, Visualization of $\mathrm{ONOO}^{-}$and viscosity in drug-induced hepatotoxicity with different fluorescence signals by a sensitive fluorescent probe, Anal. Chem., 2020, 92, 14667-14675. 
34 Y. Zhang, Z. Li, W. Hu and Z. Liu, A mitochondrial-targeting near-infrared fluorescent probe for visualizing and monitoring viscosity in live cells and tissues, Anal. Chem., 2019, 91, 10302-10309.

35 M. Fu, Y. Sun, Kenry, M. Zhang, H. Zhou, W. Shen, Y. Hu and Q. Zhu, A dual-rotator fluorescent probe for analyzing the viscosity of mitochondria and blood, Chem. Commun., 2021, 57, 3508-3511.

36 H. Qian, M. E. Cousins, E. H. Horak, A. Wakefield, M. D. Liptak and I. Aprahamian, Suppression of Kasha's rule as a mechanism for fluorescent molecular rotors and aggregationinduced emission, Nat. Chem., 2017, 9, 83-87.

37 G. Chen, W. Li, T. Zhou, Q. Peng, D. Zhai, H. Li, W. Z. Yuan, Y. Zhang and B. Z. Tang, Conjugation-induced rigidity in twisting molecules: filling the gap between aggregationcaused quenching and aggregation-induced emission, $A d v$. Mater., 2015, 27, 4496-4501.

38 R. Guo, J. Yin, Y. Ma, Q. Wang and W. Lin, A novel mitochondria-targeted rhodamine analogue for the detection of viscosity changes in living cells, zebra fish and living mice, J. Mater. Chem. B, 2018, 6, 2894-2900.

39 D. Wang, Z. Wang, X. Wang, X. Zhuang, C. Tian, F. Luan and $\mathrm{X}$. Fu, Functionalized copper nanoclusters-based fluorescent probe with aggregation-induced emission property for selective detection of sulfide ions in food additives, J. Agric. Food Chem., 2020, 68, 11301-11308.

40 J.-W. Han, M. Zuo, W.-Y. Zhu, J.-H. Zuo, E.-L. Lü and X.-T. Yang, A comprehensive review of cold chain logistics for fresh agricultural products: current status, challenges, and future trends, Trends Food Sci. Technol., 2021, 109, 536-551.

41 R. Nayak and P. Waterson, Global food safety as a complex adaptive system: key concepts and future prospects, Trends Food Sci. Technol., 2019, 91, 409-425.

42 P. T. M. Nguyen, O. Kravchuk, B. Bhandari and S. Prakash, Effect of different hydrocolloids on texture, rheology, tribology and sensory perception of texture and mouthfeel of lowfat pot-set yoghurt, Food Hydrocolloids, 2017, 72, 90-104. 